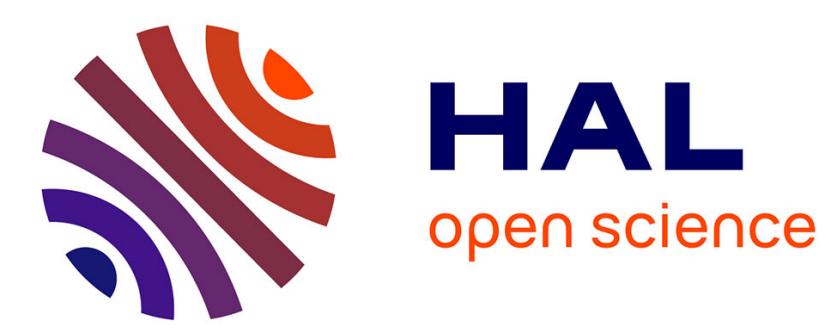

\title{
Adaptive-glasses time-domain FFOCT for wide-field high-resolution retinal imaging with increased SNR
}

Jules Scholler, Kassandra Groux, Kate Grieve, Claude Boccara, Pedro Mecê

\section{To cite this version:}

Jules Scholler, Kassandra Groux, Kate Grieve, Claude Boccara, Pedro Mecê. Adaptive-glasses timedomain FFOCT for wide-field high-resolution retinal imaging with increased SNR. Optics Letters, 2020, 10.1364/OL.403135 . hal-03020742

\section{HAL Id: hal-03020742 \\ https://hal.sorbonne-universite.fr/hal-03020742}

Submitted on 24 Nov 2020

HAL is a multi-disciplinary open access archive for the deposit and dissemination of scientific research documents, whether they are published or not. The documents may come from teaching and research institutions in France or abroad, or from public or private research centers.
L'archive ouverte pluridisciplinaire HAL, est destinée au dépôt et à la diffusion de documents scientifiques de niveau recherche, publiés ou non, émanant des établissements d'enseignement et de recherche français ou étrangers, des laboratoires publics ou privés. 


\title{
Adaptive-glasses time-domain FFOCT for wide-field high-resolution retinal imaging with increased SNR
}

\author{
Jules Scholler, ${ }^{1,2}$ (i) Kassandra Groux, ${ }^{1,2}$ Kate Grieve, ${ }^{2}$ Claude Boccara, ${ }^{1,2}$ and \\ Pedro Mecét, ${ }^{1,2, *}$ (1) \\ ${ }^{1}$ Institut Langevin, ESPCI Paris, CNRS, PSL University, 1 Rue Jussieu, 75005 Paris, France \\ ${ }^{2}$ Paris Eye Imaging Group, Quinze Vingts National Ophthalmology Hospital, Paris, France \\ *Corresponding author: pedro.mece@espci.fr
}

Received 20 July 2020; revised 15 September 2020; accepted 15 September 2020; posted 17 September 2020 (Doc. ID 403135); published 16 October 2020

\begin{abstract}
The highest three-dimensional (3D) resolution possible in in vivo retinal imaging is achieved by combining optical coherence tomography (OCT) and adaptive optics. However, this combination brings important limitations, such as small field-of-view and complex, cumbersome systems, preventing so far the translation of this technology from the research lab to clinics. In this Letter, we mitigate these limitations by combining our compact time-domain full-field OCT (FFOCT) with a multi-actuator adaptive lens positioned just in front of the eye, in a technique we call the adaptive-glasses wavefront sensorless approach. Through this approach, we demonstrate that ocular aberrations can be corrected, increasing the FFOCT signal-to-noise ratio (SNR) and enabling imaging of different retinal layers with a $3 \mathrm{D}$ cellular resolution over a $5^{\circ} \times 5^{\circ}$ field-of-view, without apparent anisoplanatism. () 2020 Optical Society of America
\end{abstract}

https://doi.org/10.1364/OL.403135

Provided under the terms of the OSA Open Access Publishing Agreement

Optical coherence tomography (OCT) revolutionized ophthalmology in the 1990s owing to its high axial resolution, which enabled clinicians to distinguish the retinal layers in vivo [1]. Although the axial resolution of OCT is sufficient to resolve retinal features at a micrometer scale, the lateral resolution is limited by ocular aberrations [2]. Owing to its capacity to correct for ocular aberrations in real-time, adaptive optics $(\mathrm{AO})$ has become the primary technique to achieve high lateral resolution in the retina [3]. When coupled to OCT, AO has enabled micrometer resolution in all spatial dimensions for in vivo retinal imaging [4], contributing to the understanding of retinal function and diseases. Nevertheless, the high lateral resolution achieved with $\mathrm{AO}$ comes with a cost of a small field-of-view (FOV), which is limited by the isoplanatic patch of the eye (around $2^{\circ} \times 2^{\circ}$ ) [5] but also by the trade-off between the spatial sampling of the scan and the acquisition speed, in order to avoid image distortion due to eye motion. Most importantly, AO-OCT systems are complex and cumbersome, requiring long imaging sessions to acquire a large FOV [4]. These limitations have prevented the translation of AO-OCT from the research lab to clinics. Much recent work has aimed at addressing these limitations, by reducing the AO-OCT system complexity and footprint through the use of conjugated lens-based wavefront sensorless $\mathrm{AO}$ [6], or by increasing the FOV to $4^{\circ} \times 4^{\circ}$ using multi-conjugate $\mathrm{AO}$ [7]. However, the former still presents a small FOV because of the limited isoplanatic patch of the eye and spatial sampling of the scan, and the latter adds complexity as two deformable mirrors are necessary.

In this Letter, we propose a lens-based sensorless $A O$ approach using a multi-actuator adaptive lens (MAL, Dynamic Optics srl, Italy) positioned in front of the examined eye, i.e., without strict pupil conjugation, in a technique we call the adaptive-glasses approach. The proposed optimization scheme does not require any calibration step and is, therefore, straightforward to implement in existing systems without increasing system footprint or optical complexity. We implemented the adaptive-glasses approach in our compact time-domain fullfield OCT (henceforth, named FFOCT) system. FFOCT was found to behave differently to conventional imaging systems and Fourier-domain OCT with regards to optical aberrations. The lateral resolution of FFOCT is less affected by symmetric aberrations [8], which dominate in the eye, owing to the use of a spatially incoherent source. This interesting feature was recently highlighted for high-resolution retinal imaging over a FOV of $3.5^{\circ} \times 3.5^{\circ}$, without apparent anisoplanatism [9]. Nevertheless, although symmetrical aberrations may not be adversely affecting the resolution in FFOCT, the presence of aberrations still provokes a loss of signal-to-noise ratio (SNR). Low SNR strongly impacts the robustness of FFOCT imaging, especially at large pupil size, and prevents imaging of retinal layers other than photoreceptors $[9,10]$. Here, we show that the use of the adaptive-glasses approach to correct for ocular aberrations can considerably increase the SNR and robustness of FFOCT, enabling imaging of different retinal layers with three-dimensional (3D) cellular resolution over a $5^{\circ} \times 5^{\circ} \mathrm{FOV}$ acquired in a single shot, while retaining a compact system design. To the best of our knowledge, the presented method is the first to ally $3 \mathrm{D}$ high-resolution, wide FOV, and small 
system footprint, which are essential characteristics for clinical deployment.

The FFOCT system was described in detail elsewhere [10] and has a footprint of $50 \mathrm{~cm} \times 30 \mathrm{~cm}$ (See Visualization 1 for a mechanical drawing of the system). Importantly for this study, the FFOCT setup comprises a spectral-domain (SD) OCT channel used to track eye axial motion and drive at $50 \mathrm{~Hz}$ loop rate a fast translation stage on which the FFOCT reference arm is mounted, enabling the FFOCT to acquire en face images at a given depth in the retina. Since the phase modulation of FFOCT is performed almost randomly by the residual eye axial motion after correction [10], the brightness of FFOCT retinal images varies from one image to another, making the FFOCT signal an unreliable merit function for wavefront correction. We therefore use the brightness of the SD-OCT B-scan as a surrogate for the FFOCT SNR optimization. The FFOCT and SD-OCT channels have central wavelengths of $850 \mathrm{~nm}(30 \mathrm{~nm}$ bandwidth) and $930 \mathrm{~nm}$ (60 nm bandwidth), respectively. Since these two wavelengths are close, correcting aberrations using the brightness of SD-OCT as a merit function is also suitable for FFOCT. For both FFOCT and SD-OCT channels, the beam diameter at the pupil is $7.5 \mathrm{~mm}$. The MAL is composed of 18 actuators and can correct up to the fourth Zernike order [6]. It has a transmission of $94 \%$ in the near-infrared and a response time of less than $2 \mathrm{~ms}$. The MAL is positioned $2-3 \mathrm{~cm}$ in front of the subject's cornea, i.e., without strict pupil conjugation. It has a $10 \mathrm{~mm}$ diameter, meaning that it is large enough to avoid vignetting and resolution loss, which would occur with a smaller numerical aperture. Its diameter and position favor anisoplanatic correction through wavefront sensorless optimization similar to a pupil-conjugated scheme [11]. The coherence gate geometry was shaped to fit the retinal curvature, and dispersion was compensated using a $20 \mathrm{~mm} \mathrm{~N}$-BAK1 optical window in the sample arm [9]. The size of an individual pixel of the FFOCT camera corresponds to $1 \mu \mathrm{m}$ in the retinal plane. The SD-OCT has an A-scan rate of $36 \mathrm{kHz}$. We chose to scan over a line of $2^{\circ} \mathrm{FOV}$ with $256 \mathrm{~A}$-scans, i.e., at $140 \mathrm{~Hz}$, providing a good trade-off between acquisition speed and SNR [10]. B-scans were averaged in the lateral dimension, and used for three purposes: (1) tracking the eye axial motion for correction in real-time, (2) guiding positioning of the FFOCT coherence gate at the layer of interest, and (3) as a merit function for the wavefront optimization. The merit function can be applied for any retinal layer of interest to automatically adjust the MAL shape to favor imaging in that particular layer.

To perform the optimization, we used the DONE algorithm [12], which had three major advantages for the problem we wished to solve. First, it does not require evaluation of the merit function gradient and, hence, mitigates the MAL hysteresis by limiting the number of times that voltages are updated. Second, the DONE algorithm is very stable due to the use of regularization and is, therefore, well suited for noisy experimental data. Finally, as we use the actuator voltages as input degree of freedom, no calibration step is necessary since no modal decomposition is used. The DONE algorithm models the unknown merit function using a random Fourier expansion (RFE) $g(x)=\sum_{k=1}^{D} c_{k} \cos \left(\omega_{k}^{T} x+b_{k}\right)$ fit to the experimental data using a least squares approach. It iteratively finds a minimum of the merit function on a compact set $X \subseteq\left[V_{\min }, V_{\max }\right]^{d}$ representing each actuator voltage (where $d=18$ is the number of actuators) by updating the RFE at each new measurement,
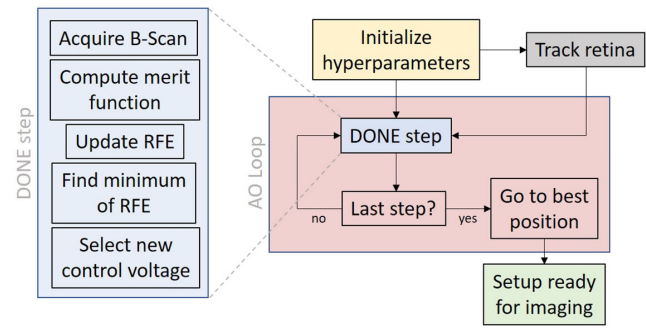

Fig. 1. Retina is tracked during the optimization to compute the merit function on the selected layer. The number of iterations was set to 50 , for a total of 1 s optimization duration.

and using this RFE as a surrogate of the merit function for optimization. Hyperparameters were selected by trial and error by imaging a model eye, and then refined for in vivo imaging. The number of basis functions $\mathrm{D}$ was set to 100 . Increasing $\mathrm{D}$ leads to a better RFE fit at the cost of more computation time (complexity is $O\left(D^{2}\right)$ ). To prevent underfitting and overfitting, a regularization parameter $\lambda$ is used in the least squares fit for finding the RFE coefficients $c_{k}$. It also helps with dealing with few measurements. We set $\lambda=0.01$. Finally, the probability density function of frequencies $\omega$ for the RFE model is drawn from a Gaussian distribution (variances $\sigma_{\zeta}^{2}=\sigma_{\xi}^{2}=1$ ) and influences the exploration of the RFE surrogate and the original function, respectively. The optimization steps are described in Fig. 1.

Retinal imaging was performed on three healthy nearemmetropic subjects (age range 25-30). Research procedures followed the tenets of the Declaration of Helsinki. Informed consent was obtained from subjects after the nature and possible outcomes of the study were explained. The study was authorized by the appropriate ethics review boards [CPP and ANSM (IDRCB number: 2019-A00942-55)]. Each subject was seated in front of the system and stabilized with a chin and forehead rest and asked to fixate a target. To maximize pupil diameter, image acquisition was performed in a dark room. Images were acquired just after wavefront optimization in parallel with real-time correction of axial eye motion. Image sequences were composed of 150 frames acquired at $300 \mathrm{~Hz}$. During image acquisition, the total power entering the eye from the FF-OCT illumination source and the SD-OCT scanning source were respectively $1.3 \mathrm{~mW}$ (for the $0.5 \mathrm{~s}$ of image acquisition) and $0.25 \mathrm{~mW}$ (continuous scanning), which are below the ocular safety limits established by the ISO standards for group 1 devices. Image processing was previously described in details elsewhere [10], and was composed of 2-phase demodulation, image selection, alignment and averaging. We used the ImageJ plugin MosaicJ to stitch together five images into a $12^{\circ} \times 12^{\circ}$ FOV. A photoreceptor density map was computed using a fully automated algorithm based on modal spacing as described in [13].

We first tested the adaptive-glasses approach on a 1951 USAF target after adding a microscope objective in the FFOCT sample arm and positioned the MAL at the back plane of the microscope objective. We added a $0.3 \mathrm{D}$ defocus in the sample arm and acquired FF-OCT images before and after correcting aberrations [Fig. 2(a)]. Aberrations induce phase artifacts yielding ringing effects and inversion of contrast. All of these were corrected after using the adaptive-glasses approach. Figure 2(b) shows the power spectral density (PSD) of these 
(a)

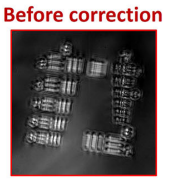

After correction

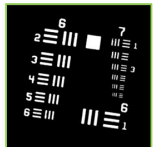

(b)

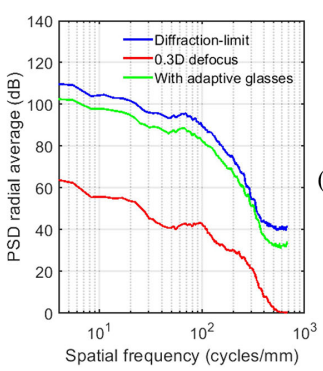

(c) Before correction

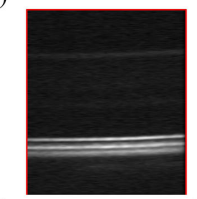

(d)

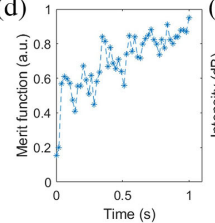

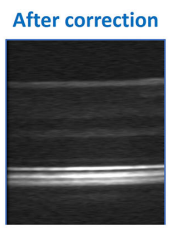

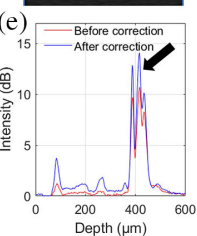

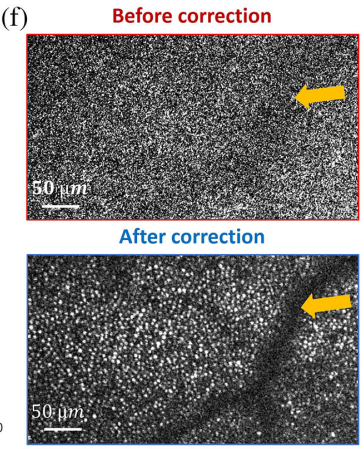

(g)

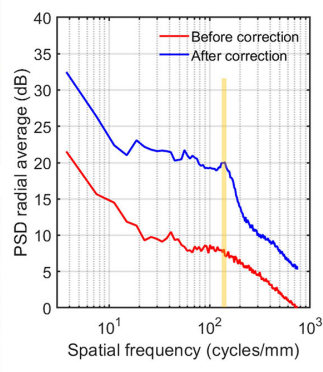

Fig. 2. Validation of the adaptive-glasses approach. (a) USAF target FFOCT image before and after aberration correction and (b) their respective PSD compared to the expected diffraction limit case. (c) SD-OCT retinal cross section before and after aberration correction. (d) Values of the merit function during wavefront optimization using the DONE algorithm. (e) Lateral average of SD-OCT B-scan highlighting the increased signal after aberration correction. Black arrow points to the retinal layer used for wavefront optimization. (f) Single non-averaged FFOCT frames before and after aberration correction at $5^{\circ}$ temporal. Image contrast was stretched independently so the shadow of a vessel positioned in a retinal layer above the photoreceptor layers can be visualized (yellow arrows). (g) Their respective PSDs, outlining the gain in terms of SNR.

images compared to the diffraction-limited PSD. Note that all three PSD plots present an almost identical distribution of spatial frequencies, showing the robustness of FF-OCT to symmetric aberrations (here defocus) in terms of resolution, while SNR drops significantly. Using the adaptive-glasses approach, we were able to recover the lost SNR, almost reaching the value we would expect for diffraction-limited imaging.

Figures 2(c)-2(g) show the capacity of the adaptive-glasses approach to correct ocular aberrations and increase the SNR at the same time for the SD-OCT [Figs. 2(c) and 2(e)] and FFOCT [Figs. 2(f) and 2(g)] for in vivo retinal imaging. The wavefront optimization [Fig. 2(d)] was realized using the signal of the cone outer segment tips [COST, black arrow in Fig. 2(e)]. Figure 2(f) presents the same retinal zone before and after correcting ocular aberrations with the adaptive glasses approach. A considerable increase in SNR is observed, thus making it possible to resolve the photoreceptor mosaic with a $7.5 \mathrm{~mm}$ pupil diameter in a single non-averaged frame. PSDs of these images, highlighting the gain in terms of SNR, are given in Fig. 2(g), where the yellow line indicates the spatial frequency of the photoreceptor mosaic, which is hidden by phase artifacts before aberration correction.

Using the SD-OCT and the axial motion stabilization, we can precisely position the FFOCT coherence gate at the retinal layer of interest. However, a mismatch of the coherence gate and focal plane positions produces low SNR FFOCT images. At the full-aperture, e.g., for a $7 \mathrm{~mm}$ pupil diameter, the depth of focus is approximately 10 times thinner than the retina, making focus position an essential step [14]. During each imaging session, SD-OCT B-scans are displayed in real-time, allowing the user to select the retinal layer of interest, where the coherence gate is then automatically positioned. In addition, when activating the wavefront optimization, only the brightness of the selected layer is taken into account, optimizing the focal plane position to the coherence gate position. Owing to this procedure, we were able to image nerve fiber layer (NFL) and photoreceptor inner/outer segment junction (IS/OS) at the same retinal region with ease (Fig. 3). Green arrows indicate the retinal layer selected for the merit function in the SD-OCT B-scan. Note that the MAL defocus amplitude correction is limited, thus limiting brightness when imaging the inner retina, which could be corrected by

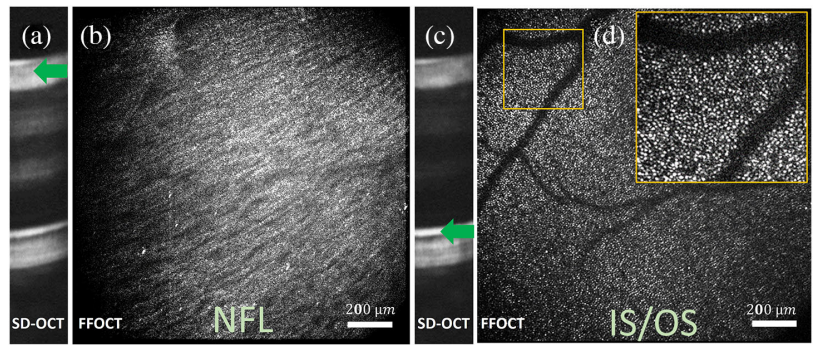

Fig. 3. Wavefront optimization for a given retinal depth. (a) and (b) SD-OCT retinal cross section and FF-OCT en face image acquired when optimizing for the retinal NFL (green arrow). (c) and (d) At the same region as (a) and (b) but at a different retinal depth after applying the wavefront optimization at the IS/OS junction.

adding a variable focal length lens [6]. Visualization 2 presents FFOCT images acquired at different depths in the NFL at $8^{\circ}$ nasal, highlighting the high axial resolution afforded by FFOCT technique (i.e., $8 \mu \mathrm{m}$ ).

Another benefit of correcting ocular aberrations for FFOCT is increased robustness. Indeed, FFOCT images are generated after a two-phase demodulation step, meaning that they carry an amplitude signal modulated by the phase difference of two consecutive images, i.e., $A \times \cos (\Delta \phi)$ (where $\mathrm{A}$ is the amplitude signal, and $\Delta \phi$ is the phase difference of two consecutive images) [10]. Since phase modulation is performed almost randomly by the residual axial eye motion after correction [10], and ocular aberrations dampen the measured amplitude, the majority of acquired images are dominated by noise. Aberration correction restores the amplitude signal, thus increasing the number of high SNR images. Visualization 3 and Visualization 4 present an FFOCT image sequence after correcting ocular aberrations with the adaptive-glasses approach, where the NFL and photoreceptor mosaic, respectively, can be visualized in single frames and monitored over time with a $6 \mathrm{~ms}$ resolution.

One important hurdle of AO-OCT for clinical translation is the challenge of allying high-spatial resolution with a wide FOV, which is beneficial for clinical applications. The combination of FFOCT and the adaptive-glasses approach opens a new avenue to wide FOV high-resolution retinal imaging in a 

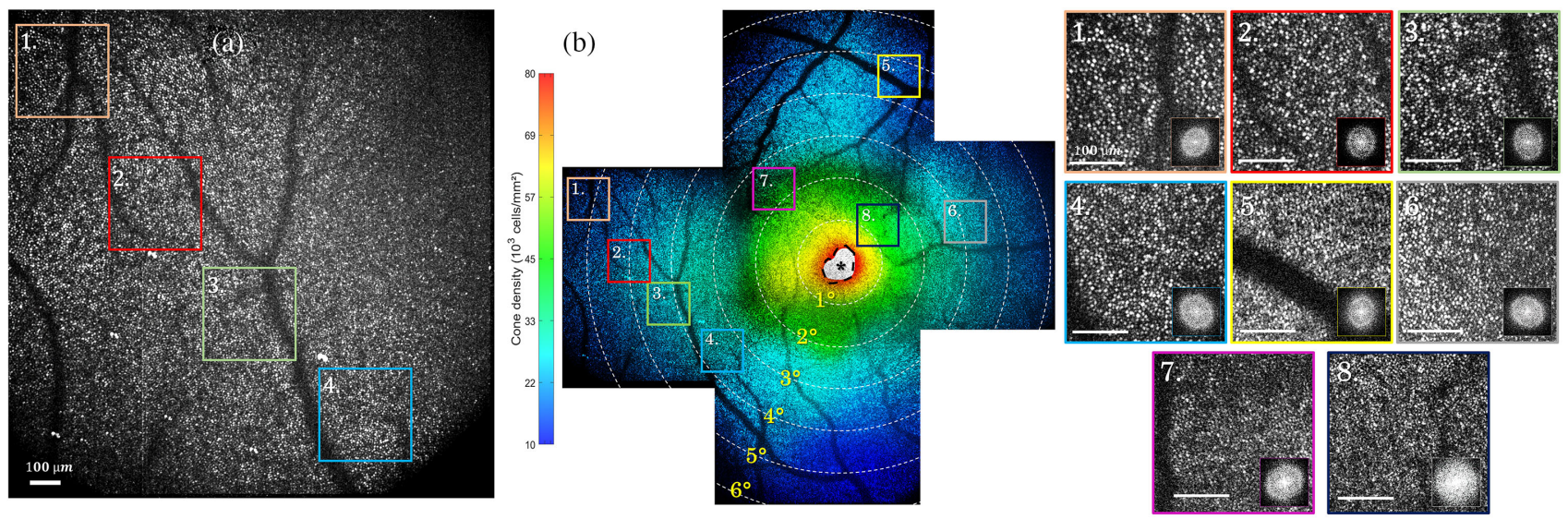

Fig. 4. (a) $5^{\circ} \times 5^{\circ}$ FOV FFOCT retinal image, where the photoreceptor can be resolved without any apparent anisoplanatism (see zoomed areas). (b) $12^{\circ} \times 12^{\circ} \mathrm{FOV}$ image after stitching together five FFOCT images. The color map represents the computed photoreceptor density. Black dashed area indicates where cone density was unreliably measured, automatically detected, and discarded. Zoomed areas of $1^{\circ} \times 1^{\circ} \mathrm{FOV}$, chosen to be representative of different eccentricities, and their respective Fourier transforms are also shown.

compact imaging system. Figure 4 (a) presents a $5^{\circ} \times 5^{\circ} \mathrm{FOV}$ image obtained in a single shot ( $0.5 \mathrm{sec}$ acquisition duration), as close as $2^{\circ}$ from the foveal center, where photoreceptors can be resolved over almost the entire FOV (limited only by the retinal curvature). Zoomed areas highlight that no apparent anisoplanatic effect is observed.

The wide FOV obtained in a single shot facilitates important tasks in the clinical environment to diagnose retinal disorders at early stages, such as image montaging and the computation of photoreceptor-based biomarkers. Figure $4(\mathrm{~b})$ shows a $12^{\circ} \times 12^{\circ} \mathrm{FOV}$ image at the fovea composed of five images acquired at different retinal locations. Photoreceptor density is color coded and was consistent with the literature [15] except within $0.5^{\circ}$ of the fovea (black dashed area) where photoreceptors were not resolved but were nevertheless automatically detected and discarded using the method proposed by [14]. The total time necessary to obtain such an image (including subject alignment, image acquisition, and processing) is about $15 \mathrm{~min}$. For comparison, an instrument with a $2^{\circ} \times 2^{\circ}$ FOV (i.e., the typical size of the eye's isoplanatic patch) would need to stitch around 60 images to obtain the same image area with an image processing time multiplied by at least a factor of 10 .

We proposed the adaptive-glasses approach as a wavefront sensorless AO method favoring small footprint and low optical complexity. We successfully applied this approach to in vivo retinal imaging using FFOCT, achieving 3D high-resolution images over a $5^{\circ} \times 5^{\circ} \mathrm{FOV}$ at $300 \mathrm{~Hz}$ in a single shot. To the best of our knowledge, this is the first demonstration of AO successfully coupled to FFOCT for retinal imaging. Although we mainly illustrated the proposed approach for dual-channel SDOCT and FFOCT retinal imaging, it can be adapted to other imaging modalities and samples. Finally, the combination of the adaptive-glasses approach with FFOCT tackles those challenges that have so far prevented transfer of AO-OCT technology from bench to clinics.

Funding. Fondation Visio; Agence Nationale de la Recherche (IHU FOReSIGHT ANR-18-IAHU-0001); Fondation Voir et Entendre (x16-CARN- 0029-01); Conseil Régional, Île-de-France (Sesame 4D-EYE EX047007); Centre
National de la Recherche Scientifique (Demeter); European Research Council (610110).

Acknowledgment. Michel Paques, José Sahel, and Mathias Fink for their clinical and technical support.

Disclosures. The authors declare no conflicts of interest.

\section{REFERENCES}

1. D. Huang, E. A. Swanson, C. P. Lin, J. S. Schuman, W. G. Stinson, W. Chang, M. R. Hee, T. Flotte, and K. Gregory, and C. A. Puliafito, Science 254, 1178 (1991).

2. J. Jarosz, P. Mecê, J.-M. Conan, C. Petit, M. Paques, and S. Meimon, Biomed. Opt. Express 8, 2088 (2017).

3. E. Gofas-Salas, P. Mecê, C. Petit, J. Jarosz, L. M. Mugnier, A. M. Bonnefois, K. Grieve, J. Sahel, M. Paques, and S. Meimon, Appl. Opt. 57, 5635 (2018).

4. R. S. Jonnal, O. P. Kocaoglu, R. J. Zawadzki, Z. Liu, D. T. Miller, and J. S. Werner, Investig. Ophthalmol. Vis. Sci. 57, OCT51 (2016).

5. P. Bedggood, M. Daaboul, R. Ashman, G. Smith, and A. Metha, J. Biomed. Opt. 13, 024008 (2008).

6. H. R. G. W. Verstraete, M. Heisler, M. J. Ju, D. Wahl, L. Bliek, J. Kalkman, S. Bonora, Y. Jian, M. Verhaegen, and M. V. Sarunic, Biomed. Opt. Express 8, 2261 (2017).

7. E. Brunner, M. F. Shirazi, M. Laslandes, W. Drexler, A. Pollreisz, C. K. Hitzenberger, and M. Pircher, Investig. Ophthalmol. Vis. Sci. 61, 224 (2020).

8. U. Tricoli and R. Carminati, J. Opt. Soc. Am. A 36, C122 (2019).

9. P. Mecê, K. Groux, J. Scholler, O. Thouvenin, M. Fink, K. Grieve, and C. Boccara, Biomed. Opt. Express 11, 4928 (2020).

10. P. Mecê, J. Scholler, K. Groux, and C. Boccara, Biomed. Opt. Express 11, 492 (2020).

11. J. Mertz, H. Paudel, and T. G. Bifano, Appl. Opt. 54, 3498 (2015).

12. L. Bliek, H. R. G. W. Verstraete, M. Verhaegen, and S. Wahls, IEEE Trans. Neural Netw. Learn. Syst. 29, 167 (2018).

13. R. F. Cooper, G. K. Aguirre, and J. I. Morgan, Transl. Vis. Sci. Technol. 8, 26 (2019).

14. P. Mecê, E. Gofas-Salas, M. Paques, K. Grieve, and S. Meimon, Biomed. Opt. Express 11, 4069 (2020).

15. C. A. Curcio, K. R. Sloan, R. E. Kalina, and A. E. Hendrickson, J. Comp. Neurol. 292, 497 (1990). 